\title{
Slow Coarsening in an Ising Chain with Competing Interactions
}

\author{
S. Redner and P. L. Krapivsky \\ Center for Polymer Studies and Department of Physics, Boston University, Boston, MA 02215, USA
}

\begin{abstract}
We investigate the zero-temperature coarsening dynamics of a chain of Ising spins with a nearestneighbor ferromagnetic and an $n^{\text {th }}$-neighbor antiferromagnetic interactions. For sufficiently large antiferromagnetic interaction, the ground state consists of $n$ consecutive up spins followed by $n$ down spins, etc. We show that the asymptotic coarsening into this ground state is governed by a multispecies reactive gas of elementary excitations. The basic elementary excitations are identified and each decays at a different power-law rate in time. The dominant excitations are domains of $n+1$ spins which diffuse freely and disappear through processes which are effectively governed by $(n+1)$-particle annihilation. This implies that the ground state is approached slowly with time, as $t^{-1 / n}$.
\end{abstract}

PACS number(s): 05.50.+q, 75.10.Hk, 82.20.Mj

\section{INTRODUCTION}

Ising models with nearest-neighbor ferromagnetic and more distant antiferromagnetic interactions exhibit rich magnetic ordering [1]. The competition between ferromagnetism and longer-range antiferromagnetism leads to different ordered states and an associated sequence of phase transitions as a function of these two interaction strengths. Such models were originally formulated to help describe the complex magnetism of the rare earths [4]. Their unusual magnetic ordering is believed to arise from the RKKY interaction [5], in which the exchange interaction between localized magnetic moments oscillates between ferromagnetic and antiferromagnetic as a function of their separation. Some of the essential consequences of this complex situation seem to be captured by Ising models with competing interactions.

One of the simplest versions is the axial next-nearestneighbor Ising (ANNNI) model in which there is an isotropic nearest-neighbor ferromagnetic interaction and a next-nearest-neighbor antiferromagnetic interaction along a single axis 2]. Even in one dimension, this system exhibits non-trivial magnetic properties. For a weak antiferromagnetic interaction, the ground state is ferromagnetic, while for a strong antiferromagnetic interaction there is an "antiphase" ground state which consists of 2 spins up, followed by 2 spins down, etc. For a specific ratio of these two interactions, an infinitely degenerate ground state arises in which each spin domain is of length 2 or greater 6.

Given the very different natures of these ground states, one might expect that dynamical behavior is also strongly affected by such competing interactions. Our goal is to understand the kinetics of an Ising chain with nearestneighbor ferromagnetic interaction $J_{1}$ and $n^{\text {th }}$-neighbor antiferromagnetic interaction $-J_{n}$, when the system is also endowed with single spin-flip Glauber dynamics. The Hamiltonian of the system is

$$
\mathcal{H}=-J_{1} \sum_{i} s_{i} s_{i+1}+J_{n} \sum_{i} s_{i} s_{i+n} .
$$

For $J_{n}>J_{1} / n$, the ground state is a sequence of $n$ consecutive up spins followed by $n$ down spins, etc. Our basic result is that for $J_{n}>J_{1}$ the asymptotic approach of the system to this alternating ground state can be described in terms of a reactive gas of elementary excitations. The rate limiting step of the coarsening is governed by the $(n+1)$-particle annihilation of the dominant excitations, which implies that the system coarsens in time as $t^{-1 / n}$.

To provide a context for this work, let us recall the well-known example of zero-temperature $(T=0)$ coarsening in the Ising-Glauber chain with nearest-neighbor ferromagnetic interactions only [7]. Spin flips inside ferromagnetic domains are forbidden at $T=0$ because they cost energy, while spins at the domain interfaces can flip freely, as indicated by

$$
\begin{gathered}
-----++++++ \\
\Downarrow \\
-----+++++++
\end{gathered}
$$

since no energy cost is involved. This spin flip is equivalent to the hopping of a domain wall "excitation" which lies between the neighboring misaligned spins. Two domain walls can annihilate when they meet, a process which leads to a reduction of the energy in the system. In terms of the spins, the annihilation is equivalent to a domain which shrinks to zero size via the process

$$
\begin{gathered}
+++++-+++++ \\
\Downarrow \\
+++++++++++++.
\end{gathered}
$$

This correspondence between the ferromagnetic IsingGlauber chain and a gas of domain walls which undergo nearest-neighbor hopping and single-species two-particle annihilation provides a simple way to understand the $t^{-1 / 2}$ coarsening dynamics of the spin system [8]. In the following sections we show that the Ising-Glauber chain with competing interactions can be understood through a similar picture of reactive excitations. 


\section{SECOND-NEIGHBOR INTERACTION}

\section{A. Strong Antiferromagnetism: $J_{2}>J_{1}$}

Let us now study the Ising-Glauber chain with competing interactions at $T=0$. To be concrete, consider first the case $n=2$, i.e., a near-neighbor ferromagnetic interaction $J_{1}$ and a second-neighbor antiferromagnetic interaction $-J_{2}$. We focus on the case of strong antiferromagnetic interaction, $J_{2}>J_{1}$, as this is the case which leads to interesting dynamics. Starting from an initial ferromagnetically ordered up state for simplicity, this system evolves to the $\ldots++--++--\ldots$ ground state by a two-stage process. First, there is an initial nucleation of down domains within regions of up spins. Within a ferromagnetically ordered region, there is an energy loss $\Delta E=4 J_{1}-4 J_{2}$ when a single spin flips to create an isolated down spin. This same energy loss arises for any nucleation event which occurs within a domain of length $\geq 5$ when the flipped spin is at least two lattice spacings from both domain walls. After this nucleation, the single-spin domain can grow to length 2 with an energy loss $\Delta E=-4 J_{2}$ as long as the length of the neighboring domain into which this inflation occurs is greater than 2. At the end of this nucleation stage, therefore, the system consists of ordered $\ldots++--++\ldots$ regions, as well as domains of size 1, 3, and 4. Further, domains of size unity exist only if both neighboring domains are of length 2 .

These remaining domains now undergo a sequence of reactions which ultimately leads to the system reaching the ground state. To determine this evolution, first consider an isolated 3-domain within an otherwise stable array to 2-domains. Since the spin on either edge of the 3-domain can flip without energy cost, the 3-domain can hop isotropically by two lattice sites, as indicated by

$$
\begin{gathered}
++--++--++-- \\
\Downarrow \\
++--+++--++--.
\end{gathered}
$$

Similarly, an isolated 1-domain within a sea of 2-domains also diffuses freely, since either neighboring spin of the 1domain can flip with no energy cost, as indicated by

$$
\begin{gathered}
--++-++-++-- \\
\Downarrow \\
--++--+--++--.
\end{gathered}
$$

These freely diffusing 1- and 3-domains are the elementary excitations of the system.

To appreciate the consequences of this statement, consider an isolated 4-domain within an otherwise stable array of 2-domains. Since there is an energy cost associated with flipping either spin in the interior of a 4-domain, this process does not occur at zero temperature. If, however, either spin at the end of the 4-domain flips, for example,

$$
\begin{gathered}
--++---++--++ \\
\Downarrow \\
--++---+++--++,
\end{gathered}
$$

then the configuration becomes two adjacent 3-domains within the stable sea of 2-domains. Each of these 3 domains can then diffuse freely; the first step of this process is indicated by

$$
\begin{gathered}
--++--+++--++ \\
\Downarrow \\
--+++--+++--++.
\end{gathered}
$$

One can thus regard an isolated 4 -domain as a $[3,3]$ resonant state which is formed whenever two 3-domains collide. This resonance is short-lived, however, since its binding energy is zero.

Continuing this reasoning, consider the evolution of a 4, 3 pair within a stable sea of 2's. Since both the 3 and 4 diffuse freely, these two excitations could move apart with zero energy cost. On the other hand, the 3-domain can shrink and the 4-domain can grow to size 5, also with zero energy cost. If this occurs, the 5 is unstable to the nucleation event: $5 \rightarrow 2+1+2$. Due to the fact that the 4 can be viewed as a $[3,3]$ resonance, the penultimate stoichiometry of this process is $3+3+3 \rightarrow 1$, i.e., 3 's annihilate through triple collisions.

Finally consider how isolated 1's evolve. Since 1's diffuse freely they react only upon meeting another 1 or a 3. In the former collision, a 4 is formed,

$$
\begin{gathered}
++--+++--++-- \\
\Downarrow \\
++--++++--++--,
\end{gathered}
$$

with an associated energy loss $\Delta E=-4 J_{1}$. Because the 4 is equivalent to a pair of 3 's, the penultimate stoichiometry of this process is $1+1 \rightarrow 3+3$. Finally if a 1 meets a 3 , they react to form a stable pair of 2 's,

$$
\begin{gathered}
++--+---++- \\
\Downarrow \\
++--++--++--.
\end{gathered}
$$

with an associated energy loss $\Delta E=-4 J_{2}$. This can be viewed as the two-species annihilation process $1+3 \rightarrow 0$, since the pair of 2's that are formed become part of the stable ground state.

The underlying stoichiometry of these processes can therefore be summarized by the three reactions

$$
\begin{array}{lll}
3+3+3 & \rightarrow 1 & \Delta E=+4 J_{1}-4 J_{2}, \\
1+1 & \rightarrow 3+3 & \Delta E=-4 J_{1}, \\
1+3 & \rightarrow 0 & \Delta E=-4 J_{2} .
\end{array}
$$

Since each of these processes leads to a loss of energy, they each occur at the same rate when $T=0$. The overall effect of these reactions is that the density of these 
elementary excitations ultimately vanish. Let us now determine the time dependence for the densities of these excitations in the mean-field limit. Using $A$ to denote both domains of length 3 and their density, and similarly using $B$ for 1 's, the rate equations associated with the reaction scheme (2) are

$$
\begin{aligned}
& \dot{A}=-3 A^{3}+2 B^{2}-A B, \\
& \dot{B}=A^{3}-2 B^{2}-A B .
\end{aligned}
$$

A naive qualitative analysis of these equations indicates that $A$ 's are asymptotically dominant. Thus using $A \gg$ $B$, the above rate equations simplify to

$$
\begin{aligned}
& \dot{A} \simeq-3 A^{3}-A B \\
& \dot{B} \simeq A^{3}-A B .
\end{aligned}
$$

Subtracting Eq. (6) from (5) gives $\dot{A}-\dot{B} \simeq-4 A^{3}$. Given $A \gg B$, we neglect $\dot{B}$ to give the closed equation $\dot{A} \simeq-4 A^{3}$. Comparing with Eq. (5) yields $A^{3} \simeq A B$. This implies $B \simeq A^{2}$, which agrees with $B \ll A$. The end results of these considerations are

$$
A(t) \simeq \frac{1}{\sqrt{8 t}} \quad \text { and } \quad B(t) \simeq \frac{1}{8 t},
$$

which are confirmed by numerical integration of the rate equations.

Let us now adapt the rate equations to the case of one dimension. Quite generally, we write

$$
\begin{aligned}
& \dot{A} \simeq-3 R-r \\
& \dot{B} \simeq R-r .
\end{aligned}
$$

Here $R$ is the rate at which $A$ 's disappear due to the triple collisions and $r$ is the rate at which both $A$ 's and $B$ 's disappear due to their mutual annihilation. These equations are at the same level of approximation as Eqs. (5) and (6), as we again neglect the effect of interactions between two $B$ 's. The rate $R$ can be shown to scale as $A^{3} / \ln (1 / A)[9]$. The cubic term is just the mean-field rate of triple collisions between $A$ 's, and the logarithmic correction arises because one dimension is the critical dimension for the three-particle reaction-diffusion processes [10]. This reaction rate gives the rate equation $\dot{A} \sim-A^{3} / \ln (1 / A)$ for three-particle annihilation. This gives the asymptotic behavior for the density $A \sim \sqrt{\ln t / t}$, which agrees with previous numerical and theoretical treatments [11]. The two-species annihilation rate $r$ can be estimated as $r \sim B / \tau$, where $\tau$ is the reaction time for an $A$ and a $B$ to meet by diffusion. This reaction time is proportional to the square of the distance between a $B$ and its nearest $A$; therefore, $\tau \sim 1 / A^{2}$ which gives $r \sim B A^{2}$.

Subtracting Eq. (9) from (8) gives $\dot{A}-\dot{B} \simeq-4 R$. Since we again anticipate that $A \gg B$, we ignore $\dot{B}$ to obtain $\dot{A} \simeq-4 R$. This relation implies that triple collisions are the dominant kinetic mechanism for elimination of $A$ 's so that this density decays as in three-particle annihilation.
The factor of 4 in $\dot{A} \simeq-4 R$ indicates that four $A$ 's eventually disappear after a triple collision - three particles are eliminated in the process $A+A+A \rightarrow B$, and then the newly-formed $B$ will eliminate another $A$. To determine $B(t)$, note that Eq. (8), together with $\dot{A} \simeq-4 R$, imply that $r \simeq R$; that is, the gain and loss terms in Eq. (9) cancel. The relation $r \simeq R$ can be rewritten as $B \sim R / A^{2} \sim-\dot{A} / A^{2}$. We therefore conclude that the density of elementary excitations are

$$
A(t) \sim \sqrt{\frac{\ln t}{t}} \quad \text { and } \quad B(t) \sim \frac{1}{\sqrt{t \ln t}} .
$$

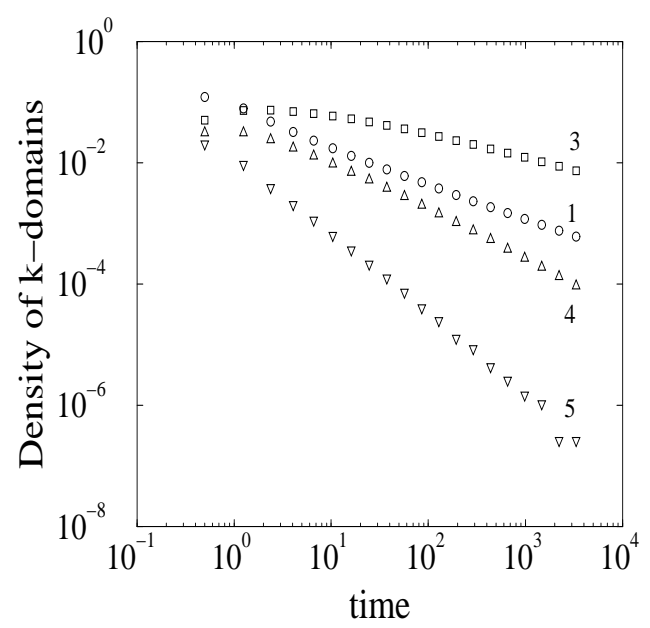

FIG. 1. Density of domains of length 1 (o), $3(\square), 4(\Delta)$ and $5(\nabla)$. Simulation results are based on 1000 realizations of a chain of 20000 sites with $J_{2} / J_{1}>1$.

Monte Carlo simulations of the $T=0$ Ising-Glauber chain yield results which are qualitatively consistent with these predictions. As shown in Fig. 1, the densities of the various elementary excitations decay at different temporal rates. Over the last 2 decades of data, linear leastsquares fits give 0.56 and 0.40 for the exponent associated with the density of 1's and 3's, respectively. However, there is considerable curvature in the data and the linear fit is not indicative of the asymptotic behavior. In fact, the data for the density of 1's is curved upward while that for the density of 3's is curved downward; these are suggestive of an asymptotic exponent of $1 / 2$. However, a serious quantitative test of Eqs. (10) would require extensive simulation, since the corrections to the local exponents will vanish only as $1 / \ln t$. We thus interpret the data as being consistent with (10). Similar least-squares fits give exponents for the density of 4's and 5's as 0.84 and 1.42. In this case, both sets of data exhibit downward curvature. These two features are qualitatively consistent with the hypothesis that 4's and 5's are equivalent to $[3,3]$ and $[3,3,3]$ resonances, respectively, so that their densities should scale as $A^{2}$ and $A^{3} / \ln (1 / A)$.

To provide an additional insight into the basic nature of this coarsening process it is worthwhile to consider the 
case $J_{1}=0$, where the chain breaks up into two independent antiferromagnetic sublattices. For each sublattice, the dynamics must coincide with the usual $t^{-1 / 2}$ Glauber coarsening. It is instructive to see how this behavior arises within our picture of elementary excitations. The crucial feature for the case $J_{1}=0$ is that the process $1+1 \rightarrow 3+3$ now involves no energy change (see Eq. (22)). Therefore, the reverse process $3+3 \rightarrow 1+1$ occurs freely. The presence of this additional process now gives the rate equations

$$
\begin{aligned}
& \dot{A}=-3 A^{3}-2 A^{2}+2 B^{2}-A B, \\
& \dot{B}=A^{3}+2 A^{2}-2 B^{2}-A B .
\end{aligned}
$$

The cubic term turns out to be negligible and the resulting asymptotic behavior is $A(t)=B(t) \sim 1 / t$. Similarly, for the one-dimensional system, the density of 1 's and 3's become identical and both decay as $t^{-1 / 2}$. The presence of this additional reaction process and the corresponding change in the dominant terms in the rate equations shows clearly that the dynamics of the Ising-Glauber chain with competing interactions is in a different universality class than that of the purely antiferromagnetic spin chain.

\section{B. Weak Antiferromagnetism: $J_{2} \leq J_{1}$}

To complete the discussion of the Ising-Glauber chain with competing interactions, let us extend the considerations discussed above to $J_{2} \leq J_{1}$. For $J_{2}<J_{1}$, the basic new dynamical feature is that nucleation of isolated domains in energetically forbidden at $T=0$. Further, any 1's in the initial population of elementary excitations quickly disappear through the annihilation processes $1+1 \rightarrow 3+3$ and $1+3 \rightarrow 0$. However 3's saturate at a finite density, as there is no mechanism for their removal. Resonances such as [3,3], [3,3,3], etc., form freely by collisions between 3's. However, high-order resonances cannot decay by nucleation but merely re-fragment into lower-order resonances.

In the case of $J_{2}<J_{1} / 2$, domain walls become the basic excitations, as in the ferromagnetic Ising-Glauber chain. However, domain walls repel since there is an energy cost $\Delta E=4 J_{2}$ to bring two domain walls closer than two lattice spacings apart. Interestingly, for all $J_{2}<$ $J_{1}$, the equilibrium ground state cannot be reached via single spin-flip Glauber dynamics. For $J_{1} / 2<J_{2}<J_{1}$, the alternating ...++ $+-++\ldots$ ground state cannot be attained because there is no mechanism for domains of length $\geq 3$ to break up; for $J_{2}<J_{1} / 2$, the ferromagnetic ground state cannot be attained because of the repulsion of domain walls.

The situations where $J_{2}=J_{1} / 2$ and $J_{2}=J_{1}$ exhibit additional new dynamical features and each requires a separate treatment. In the former case, an arbitrary array of domains which all have lengths $\geq 2$ forms a ground state [6]. The Glauber single spin-flip dynamics now has the following geometrical manifestations. First, it is energetically favorable for isolated 1-domains to disappear by "anti-nucleation". Second, domain walls can diffuse freely as long as they are more than two lattice spacings apart. They repel each other with infinite strength at zero temperature, so they cannot annihilate. Most interesting is the dynamical behavior of strings of consecutive antiferromagnetic spins. In this case, the flipping of a single spin gives rise to a 3 -domain. This can be viewed as the replacement of the triplet of antiferromagnetic spins by a ferromagnetic "trimer". This replacement process of 3 spins by a trimer can continue inside an initially antiferromagnetic region until it is converted into a ferromagnetic domain.

This dynamics is therefore equivalent to the random sequential adsorption of trimers which can overlap. The dynamics of this adsorption process can be solved according to well-known procedures [12]. Let $P_{n}(t)$ denote the probability that exactly $n$ consecutive sites are occupied by 1-domains, that is, an antiferromagnetically ordered string of length $n$. The probabilities $P_{n}(t)$ evolve according to the rate equations

$$
\dot{P}_{n}=-(n+2) P_{n}+2 \sum_{j=n+1}^{\infty} P_{j} .
$$

The loss term arises because the adsorption of a trimer whose center coincides with any of the $n$ sites of the string or the two sites adjacent to the string destroys the $n$ string. Similarly, the gain term arises from processes in which the adsorption of a trimer onto a larger string leads to the creation of an $n$-string.

To solve the rate equations, note that the ansatz

$$
P_{n}(t)=F(t) f^{n+2}(t)
$$

transforms the infinite set of differential equations (13) into the pair of equations

$$
\dot{f}=-f, \quad \dot{F}=\frac{2 F f^{2}}{1-f} .
$$

Consider now an initially disordered Ising chain (corresponding to a quench from $T=\infty$ to $T=0$ ). An antiferromagnetic string of length $n$ occurs with probability $P_{n}(0)=2^{-n-3}$, which implies

$$
F(0)=f(0)=\frac{1}{2}
$$

Solving Eqs. (15) subject to (16) yields

$$
\begin{aligned}
f(t) & =\frac{1}{2} e^{-t}, \\
F(t) & =2\left(1-\frac{1}{2} e^{-t}\right)^{2} \exp \left[e^{-t}+\frac{1}{4} e^{-2 t}-\frac{5}{4}\right] .
\end{aligned}
$$

Therefore domain walls inside antiferromagnetic strings quickly disappear and the total density of domain walls 
reaches a saturation level. Since the subsequent dynamics does not allow for processes which change the number of domain walls, the system continues to explore a sector of the phase space that contains all ground states with the same number of domain walls. The system falls into its asymptotic sector exponentially in time. Different sectors are mutually disconnected, and the sector eventually reached by the system depends on initial conditions. The dependence on initial conditions and exponential relaxation are outcomes of the lack of ergodicity in the Glauber dynamics of the Ising model with competing interactions when $J_{2}=J_{1} / 2$.

Consider finally the marginal case $J_{2}=J_{1}$. The crucial difference with the case of strong antiferromagnetism, $J_{2}>J_{1}$, is that the process $3+3+3 \rightarrow 1$ now involves no energy gain and thus the reverse process $1 \rightarrow 3+3+3$ can also occur freely. The rate equations (8) and (9) are thus modified to

$$
\begin{aligned}
& \dot{A} \simeq 3 B-3 R-r \\
& \dot{B} \simeq R-r-B
\end{aligned}
$$

To determine the asymptotic behavior, it proves convenient to transform these equations to

$$
\begin{aligned}
\dot{A}-\dot{B} & \simeq-4(R-B), \\
\dot{A}+3 \dot{B} & \simeq-4 r .
\end{aligned}
$$

We now ignore $\dot{B}$ on the left-hand sides. Combining the estimate $r \sim B A^{2}$ with Eq. (22) gives $B \sim-\dot{A} / A^{2}$. This implies $B \gg-\dot{A}$, so that Eq. (21) now gives $B \simeq R$, or $\dot{A} \sim-A^{5} / \ln (1 / A)$. We therefore conclude that when $J_{2}=J_{1}$ the density of elementary excitations are

$$
A(t) \sim\left(\frac{\ln t}{t}\right)^{1 / 4} \text { and } B(t) \sim\left(\frac{1}{t^{3} \ln t}\right)^{1 / 4} .
$$

Thus in the marginal case of $J_{2}=J_{1}$, the Ising-Glauber chain still coarsens, but at a much slower rate than in the case of strong antiferromagnetism, $J_{2}>J_{1}$.

\section{THIRD- AND MORE DISTANT-NEIGHBOR INTERACTION}

Our general approach can naturally be applied to longer range antiferromagnetic interactions. We first outline basic features of coarsening for an antiferromagnetic third-neighbor interaction; the behavior for arbitrary range antiferromagnetic interaction follows inductively. The Hamiltonian now is $\mathcal{H}=-\sum_{i}\left(J_{1} s_{i} s_{i+1}-J_{3} s_{i} s_{i+3}\right)$ and for sufficiently strong antiferromagnetic interaction the ground state consists of alternating domains of length $3, \ldots+++---+++\ldots$.. Starting from the ferromagnetic up state, the evolution to the ground state again proceeds by a two-stage process when $J_{3}>J_{1}$. While the ground state occurs when $J_{3}>J_{1} / 3$, we shall employ the stronger inequality $J_{3}>J_{1}$ in the following to guarantee that this ground state is accessible via single spin-flip Glauber dynamics.

In the initial nucleation stage, it is energetically favorable for a spin within an large up domain to flip if this spin is three or more lattice spacings from any domain boundary. Thus domains of length $\geq 7$ are unstable to such nucleation events. It is also energetically favorable for this isolated down spin domain to grow to size 3, as long as the expanding domain wall remains at least 3 lattice spacings from adjacent domain walls. At the end of the nucleation stage, therefore, all domains have length $\leq 6$. Further, domains of length 1 or 2 must be surrounded by domains of length 3 ; otherwise the central domain would expand until its size reached 3 .

A basic observation is that the true elementary excitations are domains of length 2 and of length 4 , as these are the only objects which diffuse freely within a stable sea of 3's. All other defects are resonant states of these two elementary excitations. To determine the nature of the coarsening, first consider the resonances of 4-domains. For example, a 5-domain is formed when two 4's meet and interact, so that one 4 shrinks to length 3 , while the other grows to length 5 . There is no energy cost associated with this process, so that a 5 -domain can be viewed as a $[4,4]$ resonance. Similarly, a 6 -domain is a $[5,4]$, or equivalently a $[4,4,4]$ resonance. Finally, a 7 -domain may be produced by the conversion of $6+4 \rightarrow 7+3$; thus the 7 is a $[4,4,4,4]$ resonance. At the center of the 7 -domain, a single spin can flip, thereby nucleating a 1-domain and two surrounding stable 3 domains. The penultimate stoichiometry of this process is therefore, $4+4+4+4 \rightarrow 1$.

Conversely, consider the resonances and interactions associated with 2-domains. When two 2's, within a stable sea of 3's, meet, a 1-domain is formed as indicated by

$$
\begin{gathered}
+++--++---+++ \\
\Downarrow \\
+++---+--+++.
\end{gathered}
$$

Since there is no energy cost associated with this process, a 1 -domain is simply a $[2,2]$ resonance. When three 2 's meet, there are several possible zero-energy-cost outcomes. If the interior spin of the outer 2-domain flips, then the result is $2+2+2 \rightarrow 1+3+2$. Since the 1 -domain is a $[2,2]$ resonance, this process can be considered as the first step in separating the three initial domains. However, if one of the spins in the middle domain flips, then the outcome is $2+2+2 \rightarrow 3+1+2$. Once a $3,1,2$ state is reached, it is energetically favorable for the central isolated spin to flip thus giving $3+1+2 \rightarrow 6$, i.e., a $[4,4,4]$ resonance. This last step is accompanied by the energy loss $-4 J_{1}$. The outcome of more than three 2's meeting can be obtained by grouping the 2 's into triplets and analyzing the outcome of each triplet in series. Finally, when a 2 and a 4 meet, it is energetically favorable for $2 \rightarrow 3$ and $4 \rightarrow 3$. This can be viewed as the two-species 
annihilation $2+4 \rightarrow 0$, since the two 3's formed in the reaction belong to the stable ground state.

From these basic processes, the governing reactions for this system are

$$
\begin{array}{ll}
4+4+4+4 & \rightarrow 1 \rightarrow 2+2 \\
2+4 & \rightarrow 0 \\
2+2+2 & \rightarrow 6
\end{array}
$$

For these reactions, the associated rate equations for the density of 4's $(A)$ and 2's $(B)$ are

$$
\begin{aligned}
& \dot{A}=-4 A^{4}+3 B^{3}-A B, \\
& \dot{B}=2 A^{4}-3 B^{3}-A B .
\end{aligned}
$$

The structure of these equations is similar to the secondneighbor interaction case. Following the same reasoning as used previously, we find $A(t) \sim t^{-1 / 3}$ and $B(t) \sim t^{-1}$. Similarly, we may adapt the rate equation above to describe the system in one dimension by following the approach used to write Eqs. (8) and (9). This leads to

$$
\begin{aligned}
& \dot{A} \simeq-4 A^{4}-A^{2} B \\
& \dot{B} \simeq 2 A^{4}-A^{2} B
\end{aligned}
$$

with the asymptotic behavior $A(t) \sim t^{-1 / 3}$ and $B(t) \sim$ $t^{-2 / 3}$.

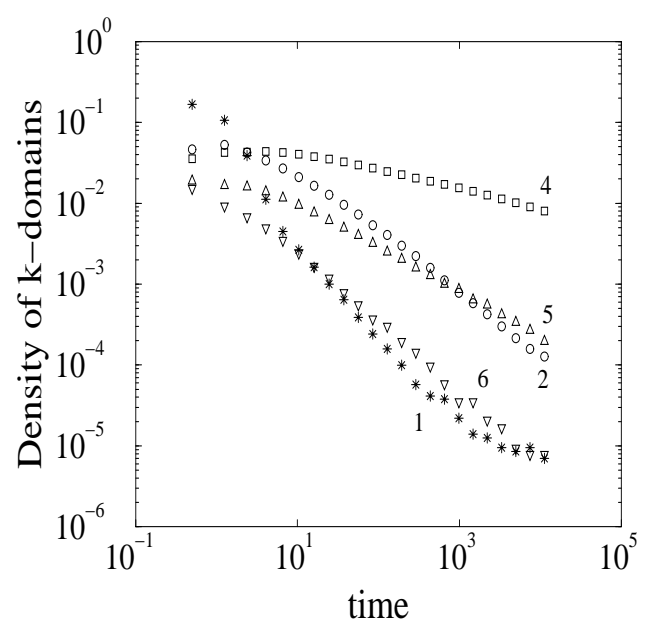

FIG. 2. Density of domains of length 1 (*), 2 (०), 4 ( $\square), 5$ $(\Delta)$, and $6(\nabla)$. Simulation results are based on 500 realizations of a chain of 20000 sites with $J_{3} / J_{1}>1$.

As in the case of the Ising chain with first- and secondneighbor interactions, simulations for the densities of the various elementary excitations indicate that they decay at different temporal rates (Fig. 2). Over the last 2 decades of data, linear least-squares fits give 0.80 and 0.25 for the exponent associated with the density of 2 's and 4's, respectively. Once again, the sense of the curvature in these two data sets is consistent with the respective asymptotic exponents of $1 / 3$ and $2 / 3$. However, even more so than in Fig. 1 the linear fit is not indicative of asymptotic behavior. We merely point out that our picture of elementary excitations allows one to express all densities in terms of the density of 4's. This predicts that the density of 1 's scales as $A^{4}$, the densities of 2 's and 5's scale as $A^{2}$, and the density of 6's scales as $A^{3}$. This is only marginally consistent with the data, a feature which we attribute to slow approach to asymptotic behavior.

Finally, we may apply a similar geometrical picture of elementary excitations to treat the general case of the Ising-Glauber chain with competing first- and $n^{\text {th }}$ neighbor interactions when $J_{n}>J_{1}$. In this case, the basic excitations are domains of $n+1$ spins $(A)$ and domains of $n-1$ spins $(B)$, each of which diffuses freely within a stable ground state sea of alternating ferromagnetic strings of length $n$. Other types of excitations are resonances of these elementary excitations. The basic kinetic mechanisms that govern the coarsening of the spin system are the $(n+1)$-particle annihilation of contiguous groups of $A$ excitations via $(n+1) A \rightarrow 1$, and the two-species annihilation $A+B \rightarrow 0$. An analysis of the corresponding rate equations again indicates that these two processes are of the same order of magnitude. Consequently, the ground state is approached as $t^{-1 / n}$ and the densities of the basic excitations decay according to $A(t) \sim t^{-1 / n}$ and $B(t) \sim t^{-(n-1) / n}$.

\section{CONCLUSIONS}

We have investigated the coarsening kinetics of the Ising chain with single spin-flip dynamics when a more distant-neighbor antiferromagnetic interaction $-J_{n}$ competes with the nearest-neighbor ferromagnetic interaction $J_{1}$. For $J_{n}>J_{1}$, this competition leads to slower zero-temperature coarsening compared to the case of nearest-neighbor ferromagnetic interactions only. Other types of non-universal relaxation phenomena have been reported for alternating interactions and other modifications of the pure Ising chain [13]. The case of the competing interaction, however, is amenable to an intuitively appealing description in terms of elementary excitations which makes clear the mechanism for the new types of coarsening kinetics. It is intriguing that the nature of the elementary excitations and the spectrum of resonances of the system are not obviously connected with the microscopic interaction of the spin system.

For general $n^{\text {th }}$-neighbor antiferromagnetic interaction, the elementary excitations are ferromagnetic strings of $n+1$ and $n-1$ spins. The former interact and disappear through $(n+1)$-particle single-species annihilation while both excitations mutually annihilate when they meet. These two processes are of the same order of magnitude so that the rate of the overall coarsening process can be viewed as being limited by $(n+1)$-particle annihilation. This leads to a coarsening which proceeds as $t^{-1 / n}$. The marginal case $J_{n}=J_{1}$ admits additional microscopic pro- 
cesses which leads to even slower coarsening. Finally, it is interesting to note that Glauber dynamics has only tenuous connection with equilibrium properties of the system. That is, the dynamical criticality at $J_{n}=J_{1}$ is disconnected from the corresponding equilibrium behavior, where ferromagnetism occurs for $J_{n}<J_{1} / n$, a ground state of alternating domains of $n$ ferromagnetic spins occurs for $J_{n}>J_{1} / n$, and an infinitely degenerate ground state consisting of alternating domains of $\geq n$ spins occurs for $J_{n}=J_{1} / n$.

We gratefully acknowledge NSF grant DMR-9632059 and ARO grant DAAH04-96-1-0114 for financial support.

[1] This model was first introduced in R. J. Elliott, Phys. Rev. 124, 346 (1961); see also S. Redner and H.E. Stanley, Phys. Rev. B 16, 4901 (1977).

[2] M. E. Fisher and W. Selke, Phys. Rev. Lett. 44, 1502 (1980) and Phil. Trans. Roy. Soc. London 302, 1 (1981); A. M. Szpilka and M. E. Fisher, Phys. Rev. Lett. 57, 1044 (1986).

[3] P. Bak and J. Von Boehm, Phys. Rev. B 21, 5297 (1980);

P. Bak, Phys. Rev. Lett. 46, 791 (1981).
[4] J. Rossat-Mignod, P. Burlet, J. Villain, H. Bartholin, T.-S. Wang, D. Florence, and O. Vogt, Phys. Rev. B 16, 440 (1977); J. Rossat-Mignod, P. Burlet, H. Bartholin, O. Vogt, and R. Lagnier, J. Phys. C 13, 6381 (1980); P. Fischer, B. Lebech, G. Meier, B. D. Rainford, and O. Vogt, J. Phys. C 11, 345 (1978); G. Meier, P. Fischer, P. Halg, B. Lebech, B. D. Rainford, and O. Vogt, J. Phys. C 11, 1173 (1978); B. Lebech, K. Clausen, and O. Vogt, J. Phys. C 13, 1725 (1980).

[5] M. A. Ruderman and C. Kittel, Phys. Rev. 96, 99 (1954); T. Kasuya, Prog. Theor. Phys. 16, 45 (1956); K. Yosida, Phys. Rev. 106, 893 (1957).

[6] S. Redner, J. Stat. Phys. 25, 15 (1981).

[7] R. J. Glauber, J. Math. Phys. 4, 294 (1963).

[8] J. G. Amar and F. Family, Phys. Rev. A 41, 3258 (1990).

[9] P. L. Krapivsky, Phys. Rev. E 49, 3233 (1994).

[10] K. Kang, P. Meakin, J. H. Oh, and S. Redner, J. Phys. A 17, L685 (1984).

[11] V. Privman and M. D. Grynberg, J. Phys. A 25, 6567 (1992); D. ben-Avraham, Phys. Rev. Lett. 71, 3733 (1993); B. P. Lee, J. Phys. A 27, 2633 (1994).

[12] For a comprehensive review, see J. W. Evans, Rev. Mod. Phys. 65, 1281 (1993); adsorption onto initially disordered substrates is described e.g. in E. Ben-Naim and P. L. Krapivsky, J. Phys. A 27, 3575 (1994).

[13] For a recent review, see S. J. Cornell, in Nonequilibrium Statistical Mechanics in One Dimension, ed. V. Privman (New York: Cambridge University Press, 1997), p. 111. 